\title{
MicroRNAs Associated with Epithelial-Mesenchymal Transition Can Be Targeted to Inhibit Peritoneal Dissemination of Human Scirrhous Gastric Cancers
}

\author{
Yoshifumi Takei $^{\mathrm{a}}$ Guodong Shen $^{\mathrm{b}}$ Ayami Morita-Kondo ${ }^{\mathrm{a}}$ Toshifumi Hara $^{\mathrm{a}}$ \\ Keichiro Miharac Kazuyoshi Yanagihara ${ }^{\mathrm{c}}$ \\ aDepartment of Medicinal Biochemistry, School of Pharmacy, Aichi Gakuin University, Nagoya, Japan; \\ ${ }^{b}$ Division of Disease Models, Center for Neurological Diseases and Cancer, Nagoya University Graduate School of \\ Medicine, Nagoya, Japan; ${ }^{C}$ Department of Hematology and Oncology, Research Institute for Radiation Biology and \\ Medicine, Hiroshima University, Hiroshima, Japan; ${ }^{\mathrm{d}}$ Division of Translational Research, Exploratory Oncology and \\ Clinical Trial Center, National Cancer Center, Kashiwa, Japan
}

\section{Keywords}

Scirrhous gastric cancer · microRNA · Peritoneal

dissemination - Epithelial-mesenchymal transition ·

Antimetastasis therapy - Nucleic acid-based medicine

\begin{abstract}
Objectives: Scirrhous gastric cancers grow rapidly, and frequently invade the peritoneum. Such peritoneal dissemination properties markedly reduce patient survival. Thus, an effective means for inhibiting peritoneal dissemination is urgently required. Methods: We previously established a cell line, HSC-58, from a scirrhous gastric cancer patient, and further successfully isolated a metastatic line, 58As9, in nude mice upon orthotopic inoculation. Using the lines, we examined the mechanism underlying peritoneal dissemination from the viewpoint of microRNA (miRNA) expression. $\boldsymbol{R} \boldsymbol{e}$ sults: miRNA array and qRT-PCR analysis showed that the expressions of epithelial-mesenchymal transition (EMT)-associated miRNAs such as miR-200c and miR-141 were significantly low in 58As9. Using 58As9 with stably overexpress-
\end{abstract}

ing miR-200c, miR-141, or both, together with a luciferase reporter assay, we found that miR-200c targeted zinc finger E-box-binding homeobox 1 (ZEB1) and miR-141 targeted ZEB2. The overexpressed lines reversed the EMT status from mesenchymal to epithelial in 58As9, and significantly reduced the invasion activity and peritoneal dissemination for a significant prolongation of survival in the orthotopic tumor models in nude mice. Conclusions: EMT-associated miRNAs such as miR-200c and miR-141 and their target genes ZEB1/ ZEB2 have good potential for antiperitoneal dissemination therapy in patients with scirrhous gastric cancers.

(c) 2018 S. Karger AG, Basel

\section{Introduction}

In the process of epithelial-mesenchymal transition (EMT), epithelial cells lose properties such as cell-cell adhesion and cell polarity, and on the other hand, they acquire more migratory and invasive properties representing mesenchymal-like cells [1-3]. During embryogenesis,

\section{KARGER}

(c) 2018 S. Karger AG, Basel

E-Mail karger@karger.com

www.karger.com/pat
Yoshifumi Takei

Department of Medicinal Biochemistry, School of Pharmacy Aichi Gakuin University

1-100 Kusumoto-cho, Chikusa-ku, Nagoya 464-8650 (Japan)

E-Mail takei@dpc.agu.ac.jp 
EMT and its reversed process mesenchymal-epithelial transition (MET) play a crucial role in the development of various organs [1-3]. EMT occurs in wound healing and organ fibrosis, and is closely associated with the initiation of metastasis, especially in cancers [1-4]. To date, EMT has generally been recognized as an important phenomenon during the process of cancer metastasis in many kinds of tumors [5-7].

Scirrhous gastric cancer, which accounts for approximately $10 \%$ of gastric cancers, is classified into poorly differentiated and diffuse-type carcinomas [8]. Scirrhous gastric cancers grow rapidly with widespread stromal fibrosis, frequently invade into the abdominal cavity, and, in many patients, metastasize to the peritoneum $[9,10]$. Although many treatment modalities have been examined, including novel chemotherapeutic-based therapy, hormonal therapy, hyperthermia therapy, and immunotherapy [11], there are currently few options for the treatment of scirrhous gastric cancers, especially with respect to the inhibition of peritoneal metastasis. Moreover, before a treatment method with particular efficacy against peritoneal metastasis can be developed, crucial data on the biological properties of scirrhous gastric cancers and the mechanisms underlying their peritoneal dissemination will be needed.

In our earlier study, we reported on an established cell line, HSC-58 [12], from a scirrhous gastric cancer patient, and further successfully isolated a metastatic line, 58As9 [13] that potently ( $100 \%$ incidence), spontaneously, and quickly induced peritoneal dissemination upon orthotopic inoculation into the stomach of nude mice. We defined HSC-58 as a parental line and 58As9 as a metastatic line $[12,13]$, and in the present study we used both lines to investigate the mechanism of peritoneal dissemination. The metastatic line 58As9 showed a spindle-shaped morphology, which is representative in cells in which EMT occurred, and we further showed that 58As9 was indeed a cell acquiring EMT properties [14].

MicroRNAs (miRNAs), a class of small noncoding RNAs, target many kinds of mRNA to induce mRNA degradation or translational inhibition [15-17]. In the present study, we investigated the relationship between the peritoneal dissemination of human scirrhous gastric cancers and EMT, by analyzing the expression of miRNAs. In 58As9, we found that the expressions of miR200c and miR-141, which are typical EMT-associated miRNAs [4], were much lower than those in HSC-58, and the differences were statistically significant. Using 58As9 cells engineered to stably overexpress miR-200c, miR141 , or both miRNAs, together with a luciferase reporter assay, we showed that miR-200c targeted zinc finger Ebox-binding homeobox 1 (ZEB1) [18], and miR-141 targeted ZEB2. The overexpressed lines showed significantly reduced invasion activity compared with the empty vector control. We also showed that the overexpressed lines showed a reduction in the peritoneal dissemination of the orthotopically inoculated tumors in nude mice, and significant prolongation of survival. Finally, we showed that synthetic miR-200c, miR-141, or both miRNAs had the potential to acquire an antimetastatic therapy effect against peritoneal dissemination in our scirrhous gastric cancer model using orthotopically inoculated 58As9 in nude mice.

\section{Materials and Methods}

\section{Cells}

We used human scirrhous gastric cancer cell lines from a patient, which were established in our earlier reports [12, 13]: HSC58 , as a parental cell established from a patient, and 58As9, as a metastatic cell isolated from the parental cells through repeated steps of orthotopic inoculation in nude mice, gathering ascites fluids from the mouse, and then reinoculation. These two cell lines were grown in RPMI 1640 medium with 10\% fetal bovine serum as described in our earlier reports $[12,13]$.

\section{RNA Isolation}

Total RNA was isolated from cells and tumor tissues using TRIzol $^{\circledR}$ (Invitrogen) according to the procedures described in our previous report [19], and the quality was analyzed by using an Agilent 2100 Bioanalyzer (Agilent Technologies) as reported previously [20]. The concentration of each total RNA was determined by using a NanoDrop ND-1000 spectrophotometer (Invitrogen).

\section{miRNA Microarray Analysis}

An miRCURY LNA ${ }^{\mathrm{TM}}$ MicroRNA Array (version 11.0; Exiqon) was used. Total RNA $(1 \mu \mathrm{g})$ was labeled with an miRCURY LNA ${ }^{\mathrm{TM}}$ MicroRNA Power Labeling Kit (Exiqon) according to the instruction manual, and then the fluorescently labeled RNA was dissolved in hybridization buffer $(350 \mu \mathrm{L})$ from the kit. The solution was incubated at $95^{\circ} \mathrm{C}$ for $2 \mathrm{~min}$, slowly cooled down to room temperature, and applied to the array slide. The slide was further incubated at $56^{\circ} \mathrm{C}$ for $16 \mathrm{~h}$, and washed with the washing buffer from the kit as described in the manual. Finally, the washed slide was dried, and scanned with a GenePix 4000B (Axon Instruments). The obtained data were normalized and analyzed using an ArrayPro Analyzer (version 4.5; Media Cybernetics) as reported previously [19].

\section{qRT-PCR for miRNAs}

The expression levels of the miRNAs were determined by TaqMan MicroRNA Assays as reported previously [19, 20]. Each total RNA (10 ng) was applied to reverse transcription with a TaqMan MicroRNA RT Kit and TaqMan MicroRNA Assay (Applied Biosystems), and the cDNA was analyzed using specific TaqMan probes (miR-200c, miR-141, miR-200b, miR-200a, and miR-429) 
and TaqMan Universal PCR Master Mix as described previously $[19,20]$. All of the reactions were performed in triplicate, and the level of each specific miRNA was normalized with the level of small nuclear RNA (RNU6B).

\section{Preparation of miRNA Stably Overexpressed Cell Lines from} 58 As9

A metastatic line, 58As9 was transfected with $1 \mu \mathrm{g}$ of hsa-miR200c or hsa-miR-141 expression vector (Takara Bio) using a Lipofectamine ${ }^{\circledR} 2000$ reagent (Invitrogen) as reported previously [19]. The transfected cells were selected with Geneticin $(200 \mu \mathrm{g} / \mathrm{mL})$ to obtain a stably overexpressed clone (58As9-miR-200c or 58As9miR-141). A stable clone overexpressed with both miR-200c and miR-141 was also prepared (58As9-miR-200c -miR-141).

\section{Inhibition Study of miR-200c, miR-141, or Both miRNAs in} HSC-58

In a parental line (HSC-58), the high expression of miR-200c and miR-141 was inhibited by the transfection of miRIDIAN MicroRNA Hairpin Inhibitor (Dharmacon, Lafayette, CO, USA). HSC-58 cells were transiently transfected with an miR-200c or miR-141 inhibitor via a cationic reagent, Lipofectamine ${ }^{\circledR}$ RNAiMAX (Thermo Scientific Co. Ltd.) according to the manufacturer's instructions. Simultaneous transfection of both the miR$200 \mathrm{c}$ and miR-141 inhibitors was also performed. The final concentration of each inhibitor in the dish was $20 \mathrm{nM}$ as described in our earlier report [19]. Each total RNA was isolated $48 \mathrm{~h}$ after the transfection and used for real-time PCR analysis.

\section{Invasion Analysis}

Invasion analysis was performed with the BD BioCoat Fluoroblok Tumor Invasion System (BD Biosciences) according to the manual. A movie provided by BD Biosciences is available to help understand and perform the procedure (https://www.jove.com/ video/1475/an-in-vitro-fluoroblok-tumor-invasion-assay). In the assay, the invaded cells toward the lower chamber through the Matrigel reagent were stained with calcein AM $(5 \mu \mathrm{g} / \mathrm{mL})$ in PBS at $37^{\circ} \mathrm{C}$ for $1 \mathrm{~h}$ as described in our earlier report [19]. Finally, the intensity of green fluorescence derived from calcein AM was measured by a plate reader with multi-detection mode (Spark ${ }^{\circledR}$; Tecan Japan, Tokyo, Japan). Thus, the fluorescence intensity directly represents the number of invading cells.

\section{In vivo Examination of Tumor Formation, Ascites,}

Disseminated Metastasis, and Survival Rate in Nude Mice

All of the animal experiments in this study were performed in compliance with the guidelines of the Institute for Laboratory Animal Research, Nagoya University Graduate School of Medicine, Aichi Gakuin University, or the National Cancer Center Research Institute. The cells (HSC-58, 58As9, 58As9-empty vector, 58As9miR-200c, 58As9-miR-141, and 58As9-miR-200c-miR-141) were orthotopically inoculated into the stomach in male BALB/c nude mice (6 weeks old; Japan SLC, Hamamatsu, Japan) as reported previously [19]. The total cell number $\left(1.5 \times 10^{6}\right.$ cells in $50 \mu \mathrm{L}$ of serum-free RPMI 1640 medium) was used to inoculate through a 29 -gauge needle. Twenty days later, the tumor formation, ascites fluids (frequency and volume), and peritoneal dissemination (omentum and mesenterium) were examined. Overall survival days in the orthotopically inoculated mice were independently determined.

miRNAs Involved in Peritoneal

Dissemination of Scirrhous Gastric Cancers

\section{Dual Luciferase Assay}

The cells (58As9-miR-200c, 58As9-miR-141, or 58As9-miR200c-miR-141) were transfected with pGL3 vector (200 ng; Promega) containing the $3^{\prime}$-UTR of ZEB1, $3^{\prime}$-UTR of ZEB1 with mutation, $3^{\prime}$-UTR of ZEB2, or $3^{\prime}$-UTR of ZEB2 with mutation. The specific base sequences of the inserted oligonucleotides are shown in online supplementary material Figure 1 (for all online suppl. material, see www.karger.com/doi/10.1159/000488801). The pGL3 vector expresses Firefly luciferase. The other vector, pRL-TK (50 ng; Promega), expressing Renilla luciferase was also co-transfected. The transfection reagent Lipofectamine 2000 (Invitrogen) was used. The cell lysates were prepared $48 \mathrm{~h}$ after the co-transfection, and finally we measured Firefly and Renville luciferase activities using dual luciferase assays (Promega) [19]. The values of each promoter activity (Firefly luciferase activity) were calculated as arbitrary units internally normalized with a Renilla luciferase reporter activity as reported previously [19].

\section{$q R T-P C R$ for Gene Expression Analysis}

The expression levels of the mRNAs for human E-cadherin (gene symbol: CDH1, Assay ID: Hs01023894_m1), N-cadherin (gene symbol: CDH2, Assay ID: Hs00362037_m1), and vimentin (gene symbol: VIM, Assay ID: Hs00185584_m1) were determined by specific TaqMan probes. HPRT1 (hypoxanthine guanine phosphoribosyl transferase1) was used for normalization [21-24].

\section{Immunoblot Analysis}

The whole cell lysates were prepared with RIPA buffer (Thermo Scientific) with a protease inhibitor mixture (Sigma), and the nuclear proteins were isolated with NE-PER Nuclear and Cytoplasmic Extraction Reagents (Thermo Scientific) as reported previously [19]. The protein concentration was determined by using a DC Protein Assay (Bio-Rad). The lysates $(20 \mu \mathrm{g})$ were separated by electrophoresis on SDS-PAGE gels and transferred electrophoretically onto nitrocellulose membranes according to our previous reports [25-28]. The following primary antibodies (Cell Signaling Japan, Tokyo, Japan) were used: rabbit anti-human E-cadherin antibody, rabbit anti-human $\mathrm{N}$-cadherin antibody, rabbit anti-human vimentin antibody, rabbit anti-human ZEB1 antibody, rabbit anti-human ZEB2 antibody, rabbit anti-human $\beta$-actin antibody, rabbit anti-human histone $\mathrm{H} 3$ antibody, and rabbit anti-human a-tubulin antibody. After the reaction with a proper secondary antibody labeled with horseradish peroxidase (Cell Signaling Japan), the peroxidase activity was detected with an enhanced chemiluminescence detection system (GE Healthcare).

\section{Immunostaining}

The immunostaining procedures have been previously reported [29]. HSC-58 and 58As9 cells were seeded onto Nunc ${ }^{\mathrm{TM}} \mathrm{Lab}^{-\mathrm{Tec}^{\mathrm{TM}}}$ Chamber Slides (4-well and glass type) and incubated overnight. The cells were fixed with $4 \%$ paraformaldehyde in PBS for 10 min. After washing, the cells were permeabilized with $0.1 \%$ Triton X-100 in PBS for $20 \mathrm{~min}$, and then the cells were blocked with $3 \%$ bovine serum albumin for $2 \mathrm{~h}$. Primary antibodies from Cell Signaling Japan against human $\mathrm{E}$-cadherin, $\mathrm{N}$-cadherin, and vimentin were used. The appropriate secondary antibodies such as Alexa-488-conjugated goat anti-rabbit IgG, or Alexa-555-conjugated goat antirabbit IgG (molecular probes) were also used. All of the photographs of the stained cells were taken using a confocal microscope system (Nikon A1 Rsi; Tokyo, Japan) as reported previously [24]. 


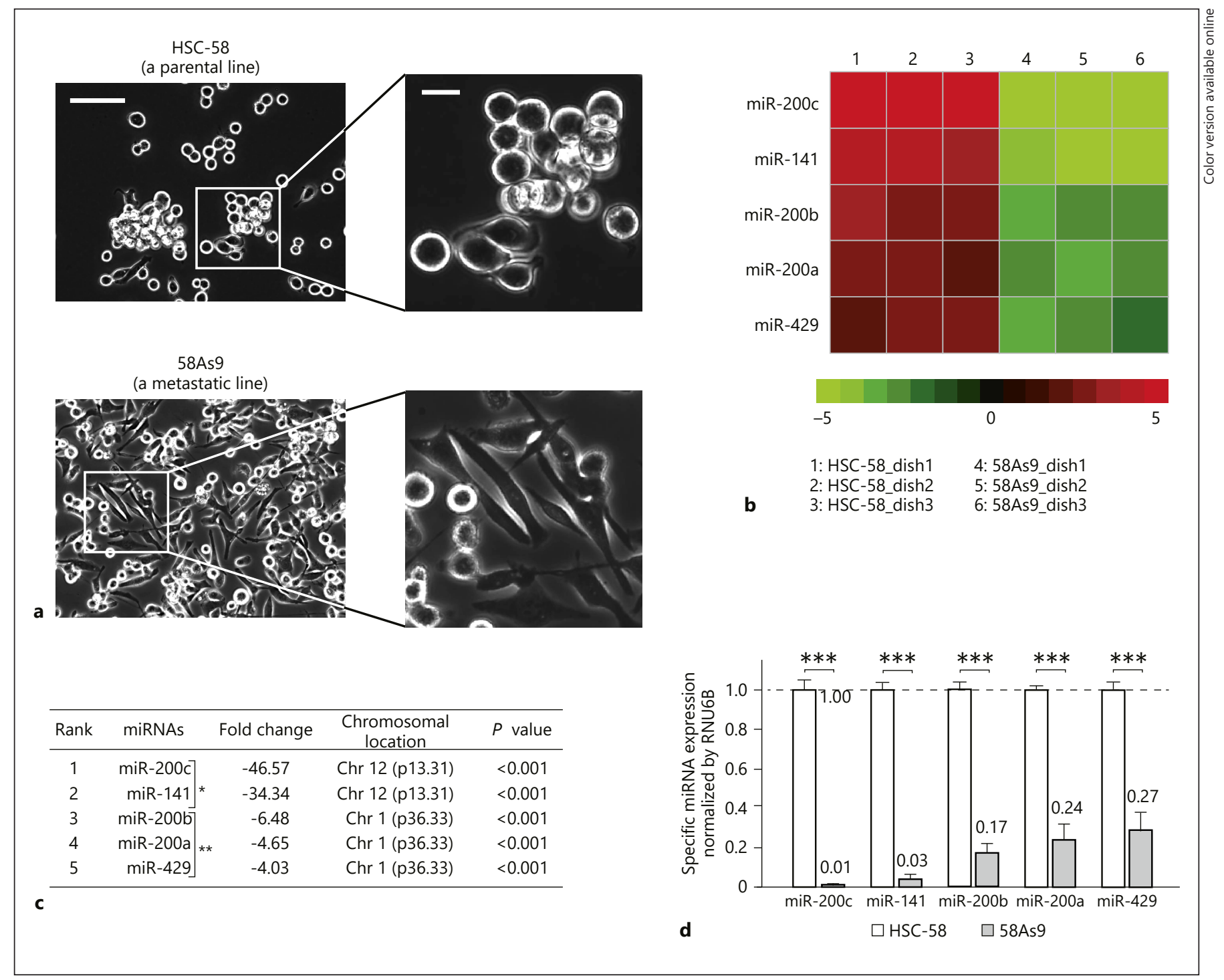

Fig. 1. miRNA array analysis and $q R T-P C R$ miRNA analysis of two human scirrhous gastric cancer cell lines, HSC-58 and 58As9. a Photographs of HSC-58 and 58As9. Scale bar, $100 \mu \mathrm{m}$. Two high magnification photographs from the original photographs are shown. The trimming areas are shown in each white box. Scale bar, $25 \mu \mathrm{m}$. b miRNA array analysis of the cell lines (lanes 1-3: HSC-58

\section{Antiperitoneal Metastasis Therapy with miRNA-Based Medicines}

The 58 As 9 cells $\left(2.0 \times 10^{6}\right.$ cells in $50 \mu \mathrm{L}$ of serum-free RPMI 1640 medium) were orthotopically inoculated into the stomach wall of nude mice. Fifteen days later, the therapy was started. Synthetic miRNAs were purchased from Dharmacon. Intravenous injections of each synthetic miRNA (control miRNA, miR-200c, miR-141, and miR-200c plus miR-141) were performed on days 0 , 1 , and 2 . All of the synthetic miRNAs $(50 \mu \mathrm{g} / \mathrm{shot})$ precomplexed with atelocollagen (Koken, Tokyo, Japan; final concentration, cells from three different culture dishes; lanes 4-6: 58As9 cells from three different culture dishes). c Top five significantly decreased miRNAs of 58As 9 compared with HSC-58. Asterisks indicate clustered miRNAs. d qRT-PCR analysis of miR-200c, miR141, miR-200b, miR-200a, and miR-429. The results are means \pm $\mathrm{SD}\left(n=4\right.$ dishes). ${ }^{* * *} p<0.001$. 


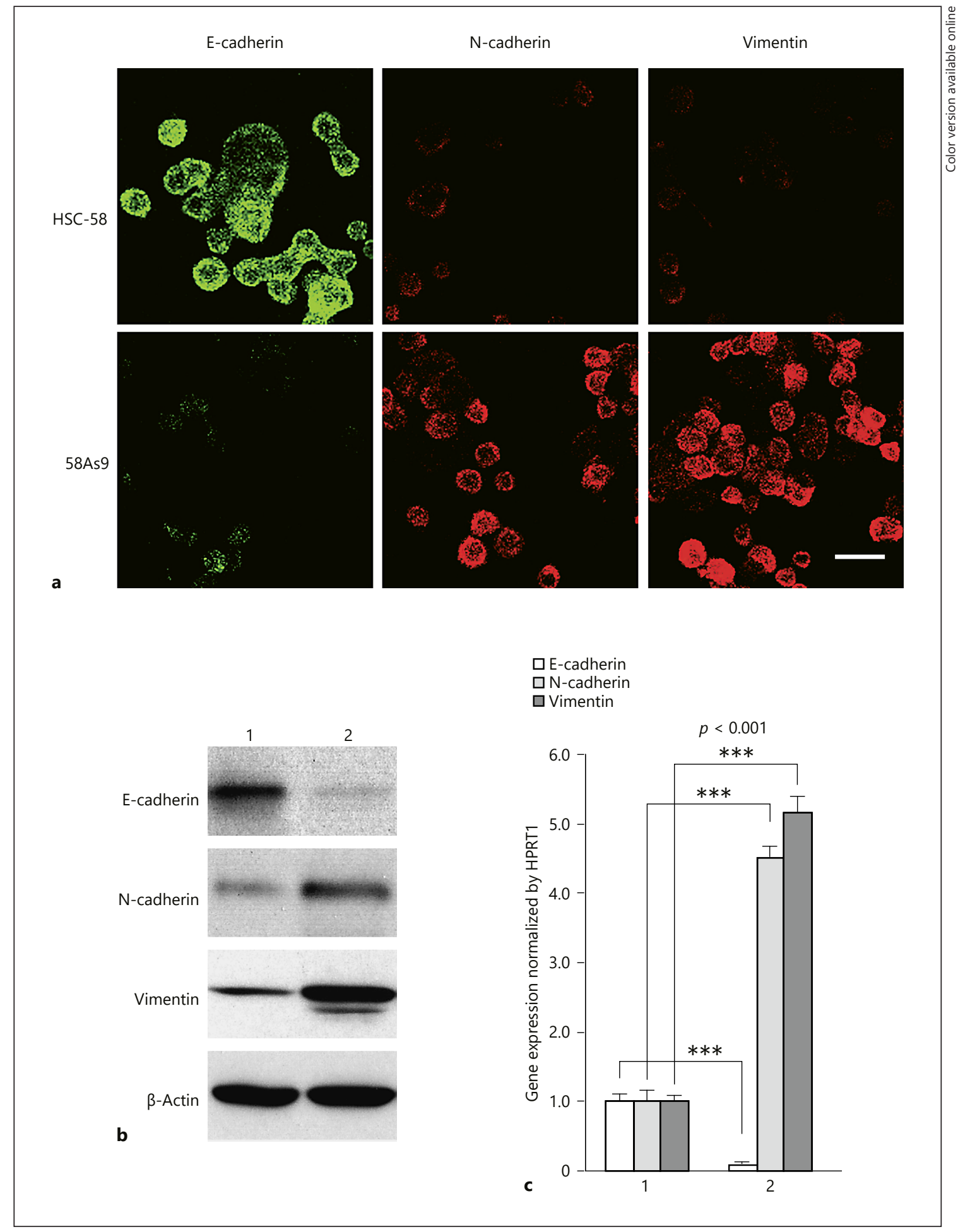

Fig. 2. Immunostaining, immunoblot, and $q R T-P C R$ analysis of HSC-58 and 58As9. a Immunostaining: E-cadherin, N-cadherin, and vimentin were stained using each specific antibody. Green fluorescence shows E-cadherin, and red fluorescence shows $\mathrm{N}$-cadherin or vimentin. Scale bar, $50 \mu \mathrm{m}$. b immunoblot analysis (lane 1 , HSC-58; lane 2, 58As9). c qRT-PCR analysis. The results are means \pm SD $(n=4$ dishes $) .{ }^{* * *} p<0.001$. 


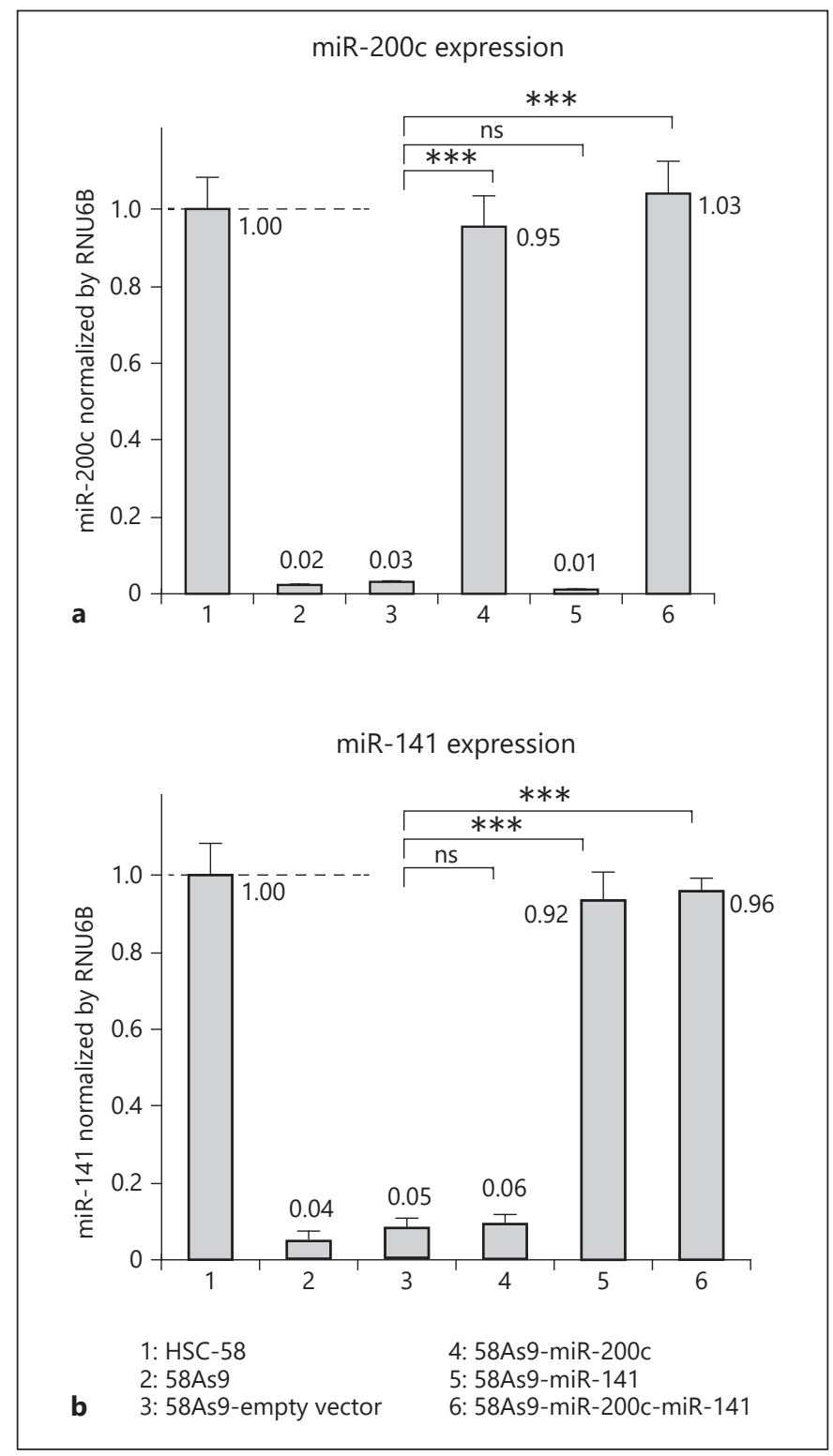

Fig. 3. Successful preparation of miR-200c, miR-141, or both miRNAs stably overexpressed 58As9 cells. a, b 58As9 cells were used as a starting material, and transfected with an miR-200c or miR141 expression vector. After the transfection, we selected positive clones using Geneticin ${ }^{\circledR}$. qRT-PCR analysis for miR-200c (a) and miR-141 (b) demonstrated the successful overexpression of both miRNAs on the 58As9 cells. The stable line, 58As9-miR-200c, was further transfected with the miR-141 expression vector to obtain a dual-miRNA-overexpressing line. The six kinds of sample names are shown in the figure. The results are means $\pm \operatorname{SD}(n=4$ dishes). *** $p<0.001$. ns, not significant.

\section{Statistical Analysis}

The statistical significance was examined by the Mann-Whitney $U$ test (nonparametric analysis). $p$ values $<0.05$ were accepted as significant.

\section{Results}

Significantly Reduced Expression of miR-200 Family Members in the Metastatic Line 58As9

To identify miRNAs involved in peritoneal dissemination of scirrhous gastric cancers, we performed a comprehensive miRNA expression array analysis using two cell lines: the parental line HSC-58 and the metastatic line 58As9. The typical morphologies of these cell lines are shown in Figure 1a. The miRNA array analysis revealed significant reductions in five miRNAs, miR-200c, miR141, miR-200b, miR-200a, and miR-429 (Fig. 1b, c) in 58 As9 compared with HSC-58 $(p<0.001)$. Two of these miRNAs showed dramatically reduced expressions (a 46fold reduction in miR-200c and a 34 -fold reduction in miR-141) in 58As9. These two miRNAs formed a cluster on chromosome 12. The other three miRNAs also formed a cluster, on chromosome 1 (Fig. 1c). Quantitative RTPCR (qRT-PCR) for the five miRNAs showed that our array results were convincing (Fig. 1d). Because many reports have described that the miR-200 family is associated with EMT [31-34], we focused on EMT in our subsequent experiments.

In the supplementary material (see online suppl. Table 1 ), we showed the other miRNA members with significantly altered expression in 58As9 compared with HSC58. In 58As9, ten miRNAs with decreased expression and ten miRNAs with increased expression were shown.

Specific Immunostaining, Immunoblot, and qRT-PCR Analysis Showed Significantly Reduced Expression of

E-cadherin and Increased Expression of N-cadherin and Vimentin in 58As9 Cells

HSC-58 cells showed potent expression with the epithelial marker E-cadherin, while almost no E-cadherin expression was seen in 58As9 cells (Fig. 2a, immunostaining; Fig. 2b, immunoblot; Fig. 2c, qRT-PCR). On the other hand, potent expression signals of mesenchymal markers such as $\mathrm{N}$-cadherin and vimentin were observed in 58 As9, and almost no expression signals of the markers were observed in HSC-58 (Fig. 2). These results indicated that our metastatic line, 58As9, exhibits mesenchymal properties.

$58 A s 9$ with Stable Overexpression of $m i R-200 c, m i R$ -

141, or Both miRNAs Was Successfully Prepared

To further investigate the relationship between EMTassociated miRNAs and peritoneal dissemination in scirrhous gastric cancers, we prepared three ectopic, stably miRNA-overexpressing cell lines from 58As9 (58As9- 


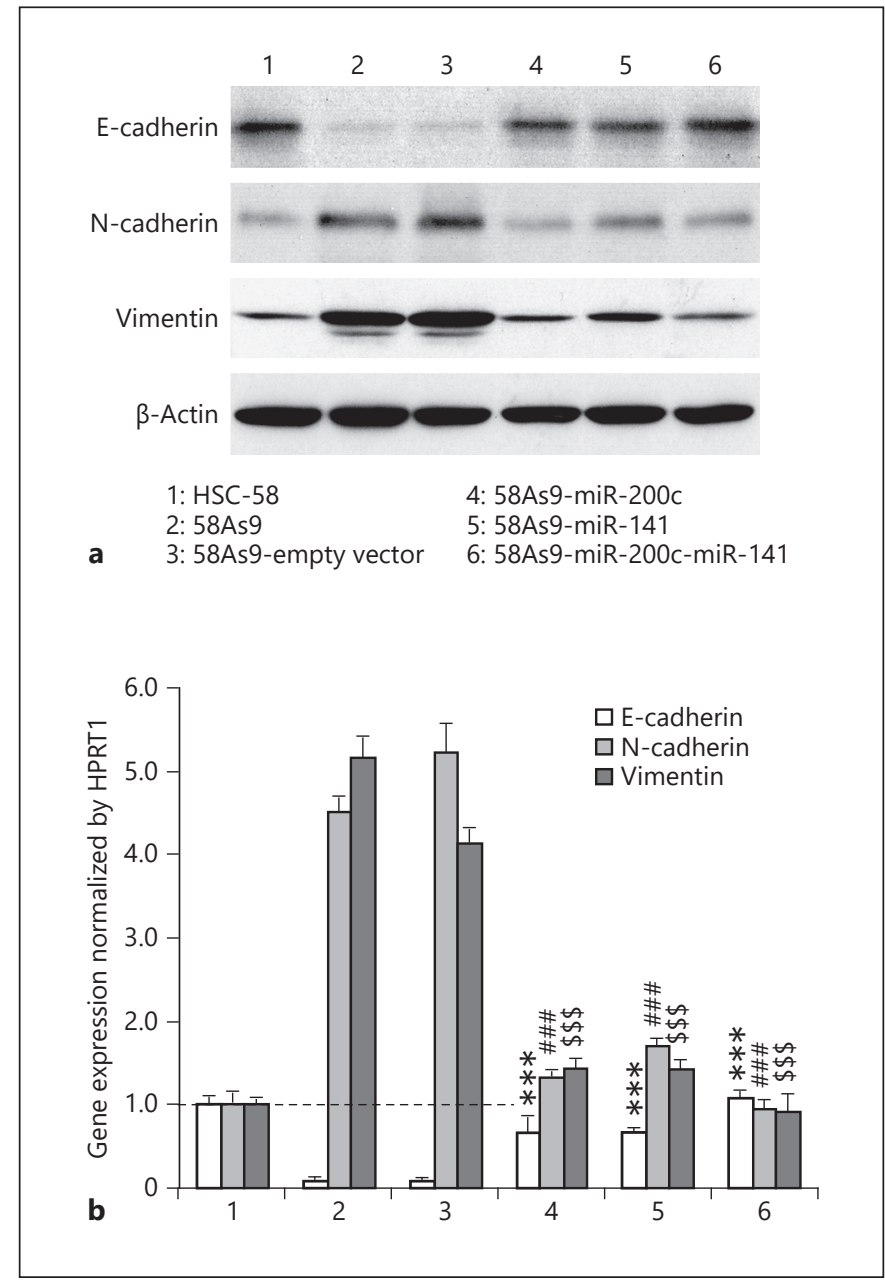

Fig. 4. E-cadherin, $\mathrm{N}$-cadherin, and vimentin expression analysis. a Immunoblot analysis. Each of the cell lysates $(20 \mu \mathrm{g} /$ lane $)$ from the six kinds of cells (lanes 1-6) indicated in the figure was subjected to SDS-PAGE and immunoblot analysis using specific antibodies. $\beta$-actin was used as a loading control. b qRT-PCR analysis. Each total RNA (800 ng) from the cells (lanes 1-6) was also reverse-transcribed, and the cDNA was analyzed using specific TaqMan probes to each gene. HPRT1 was used for normalization. The results are means $\pm \mathrm{SD}$ ( $n=4$ dishes). ${ }^{* * *} p<0.001$ versus lane 3 (E-cadherin expression in 58As9-empty vector); \#\# $p<$ 0.001 versus lane 3 ( $\mathrm{N}$-cadherin expression in 58As9-empty vector); ${ }^{\$ \$} p<0.001$ versus lane 3 (vimentin expression in the 58As9empty vector).

miR-200c, 58As9-miR-141, and 58As9-miR-200cmiR-141). qRT-PCR (Fig. 3) confirmed that all three lines stably expressed the intended miRNAs. In Figure 3a (lane 4), it can be seen that miR-200c expression was recovered to the approximate level in HSC-58 cells, while miR-141 expression level was not affected at all (Fig. 3b, lane 4). Figure $3 b$ (lane 5) shows that the miR-141 level was sim-

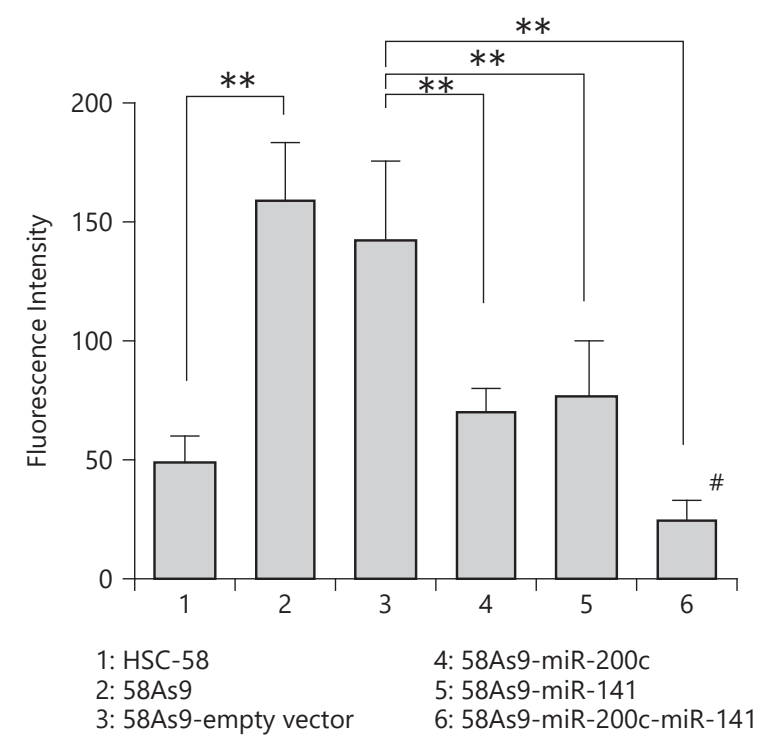

Fig. 5. Invasion analysis. The six cells were subjected to the Matrigel $^{\circledR}$ invasion assay. The invaded cells toward the lower chamber through the Matrigel reagent were stained with calcein AM (5 $\mu \mathrm{g}$ / $\mathrm{mL}$ ) in PBS at $37^{\circ} \mathrm{C}$ for $1 \mathrm{~h}$. Finally, the intensity of green fluorescence derived from calcein AM was measured by a plate reader with multi-detection mode (Spark ${ }^{\circledR}$; Tecan Japan, Tokyo, Japan). The results are means $\pm \mathrm{SD}\left(n=6\right.$ wells). ${ }^{* *} p<0.01$; ${ }^{*} p<0.05$ versus lanes 4 and 5 (58As9-miR-200c and 58As9-miR-141).

ilarly recovered to the level of HSC-58, and the miR-200c level was not affected at all (Fig. 3a, lane 5). Finally, the dual-overexpressed line of the miRNAs (58As9-miR200c-miR141) was successfully obtained (lane 6 in both Fig. 3a, b).

\section{E-cadherin Expression Was Recovered in 58As9 with Overexpression of miR-200c, miR-141, or Both miRNAs}

Using the three miRNA-overexpressing lines derived from 58As9 cells, we examined the expression of E-cadherin, N-cadherin, and vimentin (Fig. 4). The immunoblot analysis revealed a decreased expression of E-cadherin, and increased expressions of $\mathrm{N}$-cadherin and vimentin in 58As9 compared with HSC-58 (Fig. 4a, lanes 1 and 2), which was consistent with the immunostaining results in Figure 2. All three miRNA-overexpressing lines showed a recovery of E-cadherin expression relative to parental cells, and at the same time the $\mathrm{N}$-cadherin and vimentin expressions were clearly reduced (Fig. 4a, lanes 


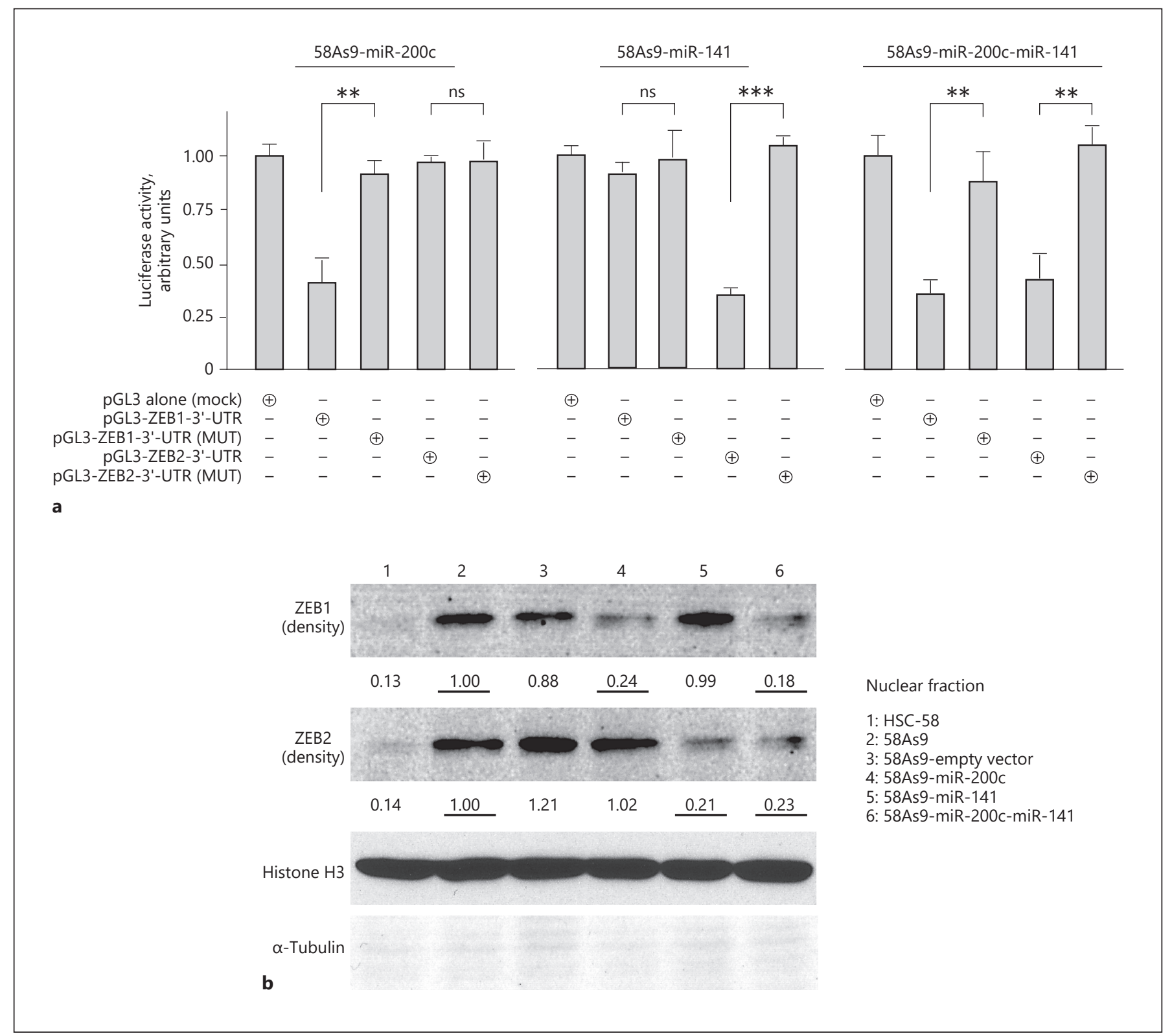

Fig. 6. miR-200c directly targeted ZEB1 and miR-141 directly targeted ZEB2 in 58As9. a Luciferase reporter assay. Three cell lines (58As9-miR-200c, 58As9-miR-141, and 58As9-miR-200cmiR-141) were transiently transfected with the five plasmids as indicated in the figure. Two days later, each cell lysate was evaluated via the dual luciferase assay. The results are means $\pm \operatorname{SD}(n=$ 6 wells). ${ }^{* *} p<0.01 ;{ }^{* * *} p<0.001$. ns, not significant. b Immuno-

4-6), suggesting that the recovered overexpression of miR-200c or miR-141 in 58As9 induced the change in cell properties from mesenchymal to epithelial. The results by qRT-PCR (Fig. 4b) using each specific TaqMan probe were similar to those by immunoblot analysis. blot analysis of ZEB1 and ZEB2. Nuclear fractions were prepared from each of six kinds of cells. The purity of the nuclear fraction was confirmed by the reactivity of histone $\mathrm{H} 3$ (a nuclear marker) and $\alpha$-tubulin (a cytoplasmic marker), respectively. ZEB1 and ZEB2 were detected by each specific antibody. The ratios of density, which were individually normalized with histone $\mathrm{H} 3$, are shown beneath the blot. The density of 58As9 was defined as 1.00 .
Invasion Activity Was Reduced in 58As9 with Stable Overexpression of miRNA-200c, miR-141, or Both miRNAs

The Matrigel invasion assay showed that the three overexpressed 58As9 lines significantly reduced invasion 
Fig. 7. The inhibition study of miR-200c and miR-141 in HSC-58. a HSC-58 cells were transiently transfected with an miR200c inhibitor, an miR-141 inhibitor, or both. After the transfection (48 h later), qRT-PCR of ZEB1 and ZEB2 was performed. The results are means \pm SD $(n=4$ dishes). ${ }^{* * *} p<0.001$. ns, not significant. b qRT-PCR analysis of E-cadherin, $\mathrm{N}$-cadherin, and vimentin. The results are means $\pm \mathrm{SD}\left(n=4\right.$ dishes). ${ }^{* *} p<0.01$.
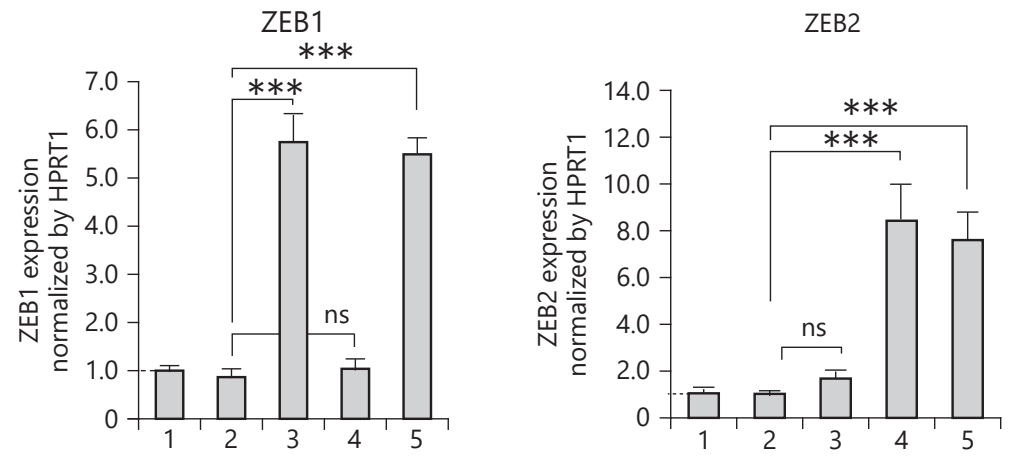

Transient transfection to HSC-58

1: Without any transfection

2: miRIDIAN microRNA Hairpin Inhibitor Negative Control \#1

3: miRIDIAN microRNA Hairpin Inhibitor for hsa-miR-200c

4: miRIDIAN microRNA Hairpin Inhibitor for hsa-miR-141

a
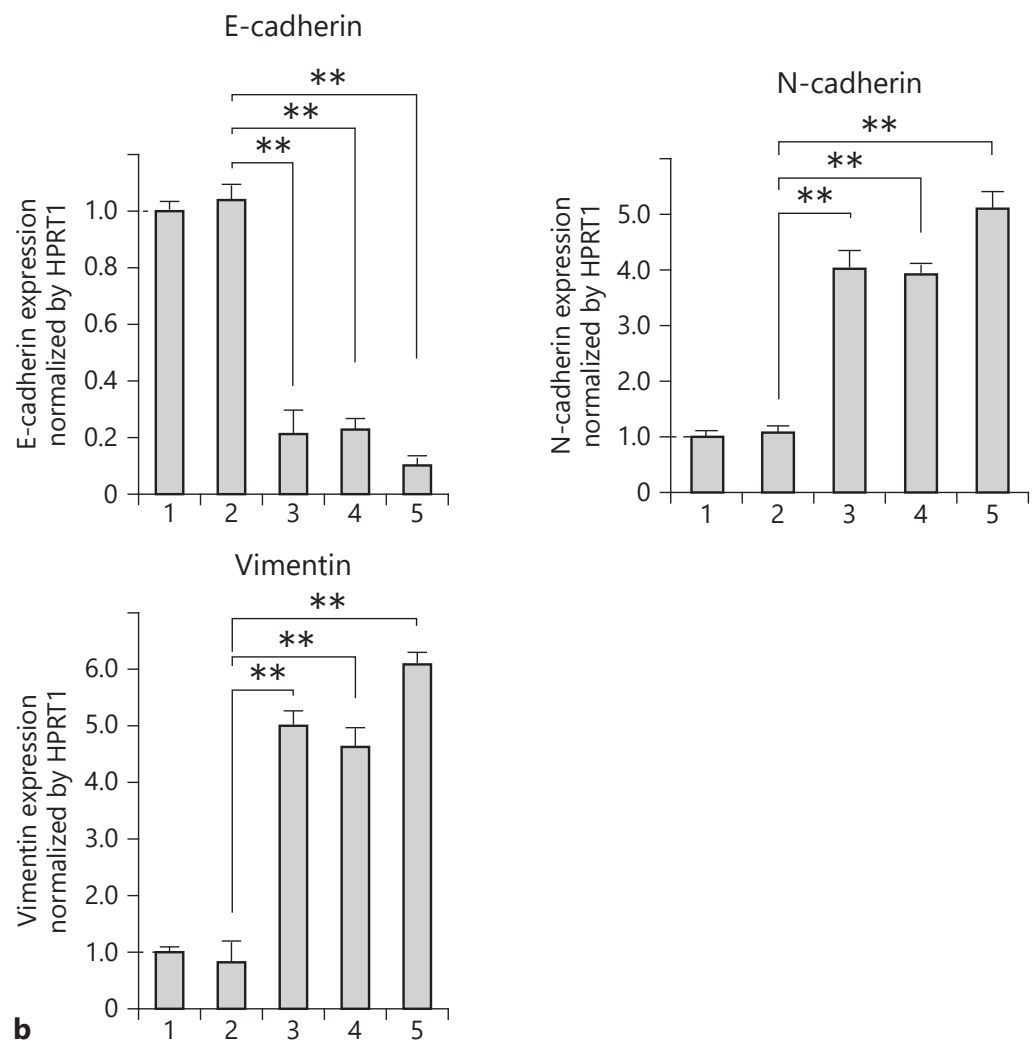

activity ( $p<0.01$; Fig. 5 ) compared with the 58As9-empty vector control. The 58As9 cells overexpressing both miRNAs exhibited a more significant inhibition effect than those overexpressing either miRNA singly $(p<0.05$;
Fig. 5). Similarly, cell migration activity as measured by transwell assay was significantly decreased by overexpression of the miRNAs (data not shown). 
Table 1. Tumor formation, ascites, and peritoneal dissemination in nude mice orthotopically inoculated with scirrhous gastric cancer cells

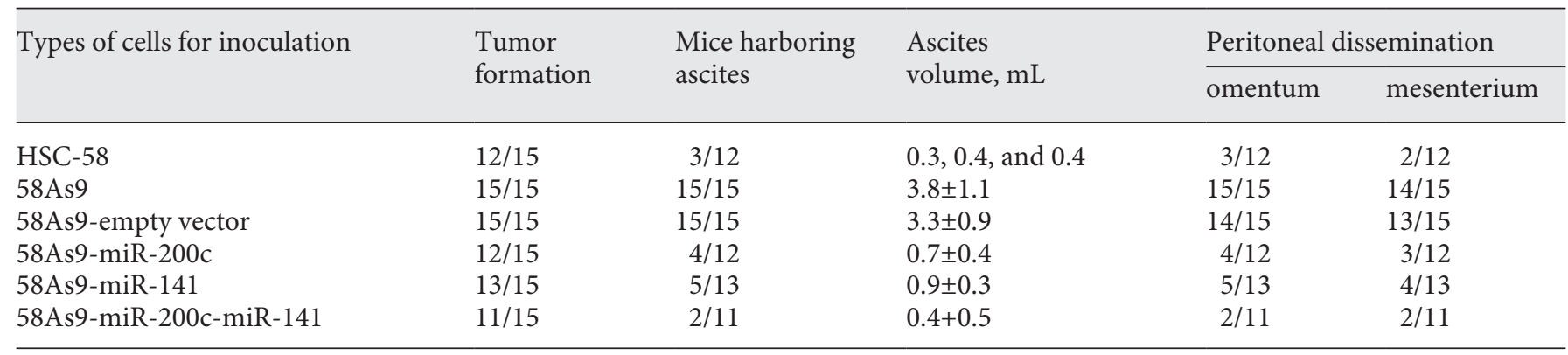

miR-200c Directly Targeted a Transcription Factor, ZEB1, and miR-141 Targeted ZEB2

During our search for a target gene of the miRNAs, a transcription factor, ZEB1, and also ZEB2 [35] showed high rank positions on miRNA target prediction programs (TargetScan Release 6.2 and miRDB online database for functional annotations). To investigate whether ZEB1 and ZEB2 were direct targets of the miRNAs in $58 \mathrm{As} 9$, luciferase reporter assays were performed based on the predicted recognition sites of the miRNAs on the 3'-UTRs of ZEB1 or ZEB2, respectively. As a result, ZEB1 was shown to be a direct target of miR-200c, and ZEB2 was also a direct target of miR-141 (Fig. 6a). The predicted base sequences for binding of the miRNAs to the $3^{\prime}$ UTRs of ZEB1 and ZEB2 were conserved in many species (e.g., humans, chimpanzees, rhesus monkeys, mice, and rats) as shown in the supplementary material (see online suppl. Fig. 1). In 58As9, the expression levels of ZEB1 and ZEB2 were high compared with HSC-58 via immunoblot analysis (Fig. 6b, lanes 1 and 2). This high expression of ZEB1 and ZEB2 in 58As9 was downregulated by the overexpression of miR-200c, miR-141, or both miRNAs (Fig. 6b, lanes 4-6), suggesting that ZEB1 and ZEB2 were direct targets of the miRNAs in 58As9.

Inhibition Studies of miR-200c or miR-141 in HSC-58

In HSC-58, we observed high expression of miR-200c and miR-141 compared with 58As9. Thus, we performed inhibition studies (knockdown studies) of miR-200c, miR-141, or both miRNAs in the cells (Fig. 7). As shown in Figure 7a, ZEB1 expression level was significantly increased via transfection of the miR-200c inhibitor, and the ZEB2 level was significantly increased via the miR141 inhibitor $(p<0.001)$. The point is that inhibition of these miRNAs induced ZEB1/ZEB2 expression in HSC58. Further, in accordance with the induction of ZEB1/
ZEB2 in HSC-58, the expression level of E-cadherin was significantly decreased ( $p<0.01$; Fig. $7 b)$. On the other hand, the levels of $\mathrm{N}$-cadherin and vimentin were significantly increased ( $p<0.01$; Fig. $7 b)$.

\section{In vivo Phenotypes of the miRNA-Overexpressing} $58 A s 9$ Lines upon Orthotopic Inoculation into Nude Mice

We orthotopically inoculated six lines (HSC-58, 58As9, 58As9-empty vector, 58As9-miR-200c, 58As9miR-141, and 58As9-miR-200c-miR-141) into the stomach wall of BALB/c nude mice using a method similar to that in our previous study [19]. Although 58As9 showed a high tumor formation ratio in nude mice (all 15 mice examined formed tumors) at 20 days after the inoculation, the ratio was slightly decreased when the mice were inoculated with cells overexpressing either or both of the miRNAs (Table 1). Regarding the ascites fluid, only 2 mice harbored ascites fluid in the body when inoculated with the cell line overexpressing both miRNAs. The actual volume $(\mathrm{mL})$ is shown in the table. The disseminated metastasis from primary tumors on the stomach wall to the omentum and mesenterium was inhibited by the overexpression (Table 1).

The number of days of survival of the inoculated mice was examined over the long term (Table 2). The mice that were simply inoculated with 58As9 died immediately (around 30 days after inoculation). In contrast, the mice inoculated with 58As9-miR-200c, 58As9-miR-141, or 58As9-miR-200c-miR-141 showed significantly prolonged survival rates (Table 2).

\section{Antiperitoneal Metastasis Therapy with Synthetic miRNA-Based Medicines}

To test the therapeutic potential of synthetic miRNAbased agents, we prepared 58As9 tumor-bearing nude
10
Takei/Shen/Morita-Kondo/Hara/Mihara/ Yanagihara 
Fig. 8. Antiperitoneal dissemination therapy with miRNA-based medicines. a The therapeutic procedures. 58As9 cells were orthotopically inoculated. Fifteen days later, various miRNA-based therapeutics (control miRNA, miR-200c, miR-141, and miR-200c plus miR-141) complexed with atelocollagen were injected into the tumorbearing mice (on days 0,1 , and 2 as indicated in the figure). The set of three i.v. injections was repeated on each of days 10 , 11 , and 12 . In each therapeutic group, 15 mice were tested. On day 20, all of the mice were sacrificed, and the primary tumor tissues were excised. b qRT-PCR for miR200c and miR-141. Using total RNA from primary tumor tissues, qRT-PCR was performed. The results are means \pm SD $(n=$ 15 mice). ${ }^{* * *} p<0.001$. ns, not significant. c qRT-PCR for human ZEB1 and ZEB2. The results are means $\pm \mathrm{SD}(n=15$ mice $)$. *** $p<0.001$. ns, not significant. d The volume of ascites fluid and peritoneal dissemination in the mice. The results (bar graphs) are means $\pm \mathrm{SD}(n=15$ mice $){ }^{*} p<0.05$; ${ }^{* *} p<0.01$; ${ }^{* * *} p<0.001$.

miRNAs Involved in Peritoneal Dissemination of Scirrhous Gastric Cancers

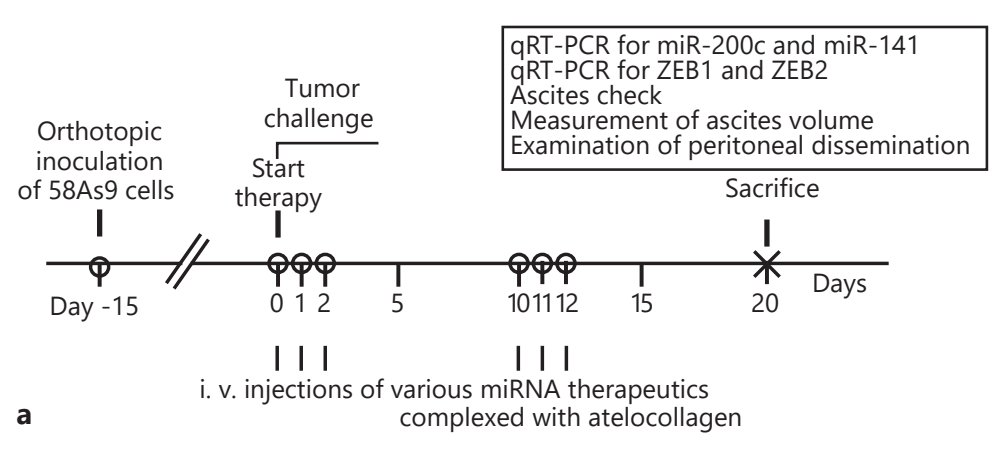

Therapy groups $\begin{array}{lll}\text { 1: } & \text { Control miRNA } \\ \text { 2:: } & \text { miR-200c } \\ \text { 3: } & \text { miR-141 } \\ \text { 4: } & \text { Combination (miR-200c + miR-141) }\end{array}$
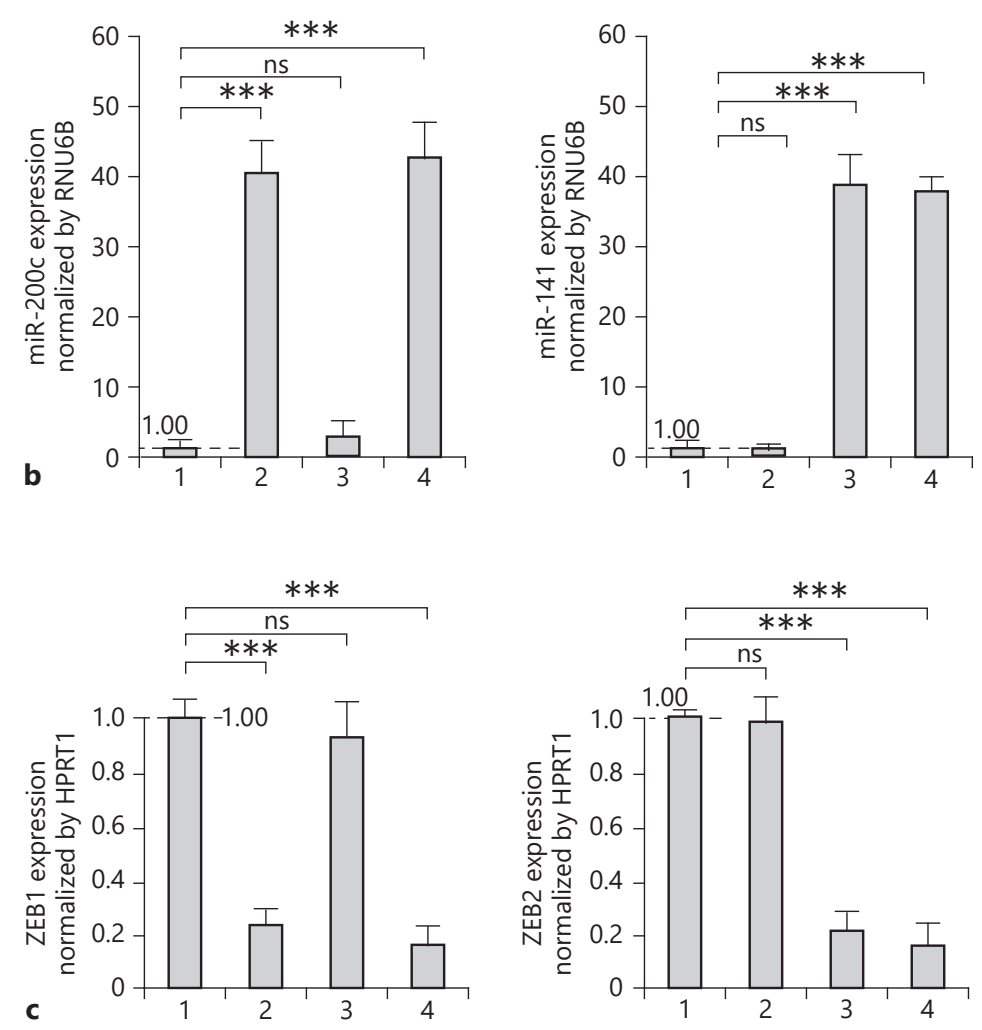

\begin{tabular}{rccc}
\hline \multirow{2}{*}{$\begin{array}{c}\text { miRNA-based } \\
\text { therapeutics }\end{array}$} & $\begin{array}{c}\text { Ascites } \\
\text { (mouse } \\
\text { numbers) }\end{array}$ & Peritoneal dissemination \\
\cline { 3 - 4 } & & Omentum & Mesenterium \\
\hline Control miRNA (1) & 15 & 15 & 14 \\
miR-200c (2) & 15 & 7 & 6 \\
miR-141 (3) & 15 & 8 & 7 \\
$\begin{array}{r}\text { Combination (4) } \\
\text { (miR-200c }\end{array}$ & 15 & 4 & 3 \\
+ miR-141) & & & \\
\hline
\end{tabular}

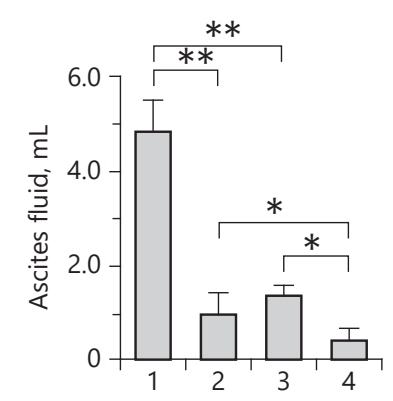

d

(1)

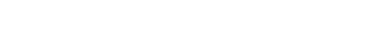


Table 2. Survival of nude mice orthotopically inoculated with scirrhous gastric cancer cells

\begin{tabular}{ll}
\hline Types of cells for inoculation & Survival, days \\
\hline HSC-58 & $96 \pm 19$ \\
58As9 & $33 \pm 8$ \\
58As9-empty vector & $39 \pm 9$ \\
58As9-miR-200c & $69 \pm 13^{* *}$ \\
58As9-miR-141 & $66 \pm 11^{* *}$ \\
58As9-miR-200c-miR-141 & $101 \pm 16^{* * *}$ \\
\hline
\end{tabular}

Fifteen mice were examined in each group. ${ }^{* *} p<0.01$; ${ }^{* * *} p<$ 0.001 versus 58As9-empty vector.

Table 3. Significantly prolonged survival using antiperitoneal dissemination therapy with miRNA-based medicines

\begin{tabular}{ll}
\hline miRNA-based medicines & Survival, days \\
\hline Control miRNA & $31 \pm 11$ \\
miR-200c & $71 \pm 12^{*}$ \\
miR-141 & $67 \pm 14^{*}$ \\
Combination (miR-200c + miR-141) & $98 \pm 18^{* *}$
\end{tabular}

Fifteen mice were examined in each therapy group. ${ }^{*} p<0.05$; ** $p<0.01$ versus control miRNA.

mice by orthotopic inoculation of the cells (Fig. 8a). Fifteen days later, synthetic miRNA-based medicines complexed with atelocollagen were intravenously injected (Fig. 8a). A biomaterial, atelocollagen (Koken) acts to deliver synthetic miRNA molecules specific to the 58As9 orthotopic tumors via intravenous injections, as we or the other group reported previously in the case of siRNA/ miRNA delivery $[21,22,36]$. On day 20 (at the end point of the therapy), all of the mice were sacrificed, and the primary tumor tissues were excised. Each total RNA isolated from the primary tumor tissues was used for qRTPCR. In the treated mice, qRT-PCR showed that the expression levels of miR-200c and miR-141 were significantly increased compared with those of the control miRNA ( $p<0.001$; Fig. $8 \mathrm{~b}$ ). Accordingly, the expressions of the target genes, ZEB1 and ZEB2, were significantly downregulated in the treated groups $(p<0.001$; Fig. $8 \mathrm{c})$. Also in the treated groups, the volume of ascites was significantly reduced, and peritoneal dissemination to the omentum or mesenterium was suppressed (Fig. 8d). Combination therapy using both the synthetic miR-200c and synthetic miR-141 showed stronger inhibitory effects on ascites and peritoneal dissemination compared with the use of either miRNA singly (Fig. 8d). Finally, our antiperitoneal dissemination therapy led to a significantly prolonged survival rate in nude mice (Table 3 ).

\section{Discussion}

Cancer metastasis causes poor clinical outcome in many patients. In particular, peritoneal dissemination in gastrointestinal and ovarian cancers dramatically shortens the survival period $[37,38]$. Peritoneal dissemination in gastrointestinal cancers is a particularly dangerous metastasis mode in which cancer cells in primary tumors break through the wall of the digestive ducts and scatter broadly into the abdominal cavity $[9,10]$. Scirrhous gastric cancers are a representative example and frequently exhibit peritoneal dissemination $[9,10]$, although there are currently few effective therapies for these malignancies. In the present study, we found miRNAs closely associated with peritoneal dissemination of scirrhous gastric cancers (Fig. 1). The five miRNAs (miR-200c, miR-141, miR-200a, miR-200b, and miR-429), which belong to the miR-200 family, were significantly reduced in the metastatic line 58As9 compared with the parental line HSC-58. Two clone lines (58As9-miR-200c and 58As9-miR-141) with stable overexpression of miR-200c or miR-141 showed reduced activities of both cell invasions (Fig. 5). The stable line with overexpression of both miRNAs (58As9-miR-200c-miR-141) showed greater reductions in these activities than the lines with overexpression of either miRNA singly (Fig. 5). These stable overexpressions of the miRNAs successfully reversed the EMT status in 58As9: in natural 58As9, the level of E-cadherin was low, and the $\mathrm{N}$-cadherin and vimentin levels were high, whereas in the miRNA-overexpressing 58As9, the levels of Ecadherin were high, and the $\mathrm{N}$-cadherin and vimentin levels were low (Fig. 2, 4). This inversion effect of the EMT status via overexpression of the miRNAs led to a suppression of the peritoneal dissemination of 58As 9 upon orthotopic inoculation into nude mice (Table 1). The overexpression also induced little ascites fluid and prolonged the survival in the orthotopic model (Tables 1,2). Finally, our results showed the feasibility of synthetic miRNA-based medicine as an antiperitoneal dissemination therapy: targeting miR-200 family members to interfere with EMT was a promising method to inhibit peritoneal dissemination of scirrhous gastric cancers (Fig. 8; Table 3).

The most significant finding of our study was that the miR-200 family associated with EMT can be targeted to
12

Pathobiology

DOI: $10.1159 / 000488801$
Takei/Shen/Morita-Kondo/Hara/Mihara/ Yanagihara 
inhibit peritoneal dissemination of scirrhous gastric cancers. We successfully showed the feasibility of miRNAbased medicine at the whole-body level by using an orthotopic tumor model in mice. Our orthotopic tumor model spontaneously induced peritoneal dissemination with perfect frequency in nude mice and thus resembled the human clinical situation. Our orthotopic tumor model was clearly different from other models such as ectopic tumor models (subcutaneous inoculation of tumors onto the back or lower flank of nude mice) or simply models using tumor cell injection into the abdominal cavity of nude mice. Such models never reflect the human clinical situation, since they tend to largely ignore various factors of the tumor microenvironment. Therefore, our approach had a significant therapeutic effect against peritoneal dissemination, and application to clinical trials could be warranted, although additional studies will be needed to confirm this.

In our metastatic line 58As9, we found that miR-200c directly targeted ZEB1 and miR-141 targeted ZEB2 via luciferase reporter assay (Fig. 6). According to recent studies [39-41], ZEB1 plays a valuable role and has clinical significance in peritoneal dissemination of gastric cancers, and is a promising target molecule for prognosis and therapy. More recently, ZEB2 has been garnering similar attention for its potential in gastric $[42,43]$ and other cancers $[44,45]$. Indeed, we found that siRNA-mediated downregulation of ZEB1 or ZEB2 successfully inhibited peritoneal dissemination in our own orthotopic 58As9 tumor models (Takei Y, et al., unpublished results). Thus, the miR-200c/miR-141 and ZEB1/ZEB2 axis regulating EMT status in tumors can be targeted to inhibit peritoneal dissemination of scirrhous gastric cancers.

Blockade of the Wnt- $\beta$-catenin pathway (canonical pathway) was also effective at inhibiting peritoneal dissemination in our previous report [19]. In that study, we used a parental cell line (HSC-44PE) from another scirrhous gastric cancer patient, and its derivative metastatic line, 44As3. In 44As3 cells, the level of miR-516a-3p was significantly reduced compared with that in the HSC$44 \mathrm{PE}$ cells, and this reduction in expression increased sulfatase1 (SULF1) expression due to a direct target gene of the miRNA [19]. The increased SULF1 released more ECM-bound Wnt ligands (Wnt3a and Wnt5a) into the medium, and through activation of the specific receptor $(\mathrm{Fz}), \beta$-catenin was translocated to the nucleus, and finally TCF/LEF was activated [19]. With respect to the treatment of 44As3-orthotopic tumors in nude mice, supplementary administration of the reduced miRNA (miR- 516a-3p) showed an antiperitoneal dissemination effect, which prolonged the survival of the treated mice [19]. Regarding the cell lines used herein (i.e., HSC-58 and 58As9), miR-516a-3p was also significantly but not dramatically reduced in 58As9 cells [19], and also directly targeted SULF1 (Takei Y, et al., unpublished results). Also in 58 As9 cells, the miR-516a-3p-SULF1- $\beta$-catenin axis made a weak contribution to peritoneal dissemination (Takei Y, et al. unpublished results). This raised a question - namely, why was peritoneal dissemination of a cell line (44As3) from a scirrhous gastric cancer patient regulated by the Wnt canonical pathway, while peritoneal dissemination of another cell line (58As9) from another patient was regulated by EMT. We have no knowledge of the molecular mechanism determining the dependence (Wnt or EMT) in the peritoneal dissemination. It is possible that Wnt and EMT exchange crosstalk during the dissemination process. In any case, in order to fully clarify the peritoneal dissemination of scirrhous gastric cancers, it is important to determine the dependence, at least dominantly, on Wnt or EMT, suggesting the necessity of individualized medicine(s).

The concept of metastasis-initiating cells (MICs) has been proposed [46-48]. MICs originate in the primary tumors and are considered a special subpopulation of circulating tumor cells in the bloodstream of cancer patients $[49,50]$. Based on the concept of MICs, the line 58As9 is a peritoneal dissemination-initiating cell. Our isolating work of 58As9 from parental HSC-58 can be regarded as work for the enrichment and amplification of peritoneal dissemination-initiating cells. To date, although little information is available for identifying peritoneal dissemination-initiating cells such as cell surface markers and their properties, we can provide a trigger concept that EMT status and EMT-associated miRNA expression should be taken into account for the identification of peritoneal dissemination-initiating cells.

\section{Conclusions}

In summary, we demonstrated that EMT-associated miRNAs such as miR-200c and miR-141 and their target genes ZEB1/ZEB2 are feasible targets for antiperitoneal dissemination therapy of human scirrhous gastric cancers.
miRNAs Involved in Peritoneal

Dissemination of Scirrhous Gastric Cancers 


\section{Acknowledgments}

We thank Drs. Kazuo Kita and Takayuki Okubo for their helpful suggestions regarding the experiments. We also thank Dr. Kuniaki Suzuki, Dr. Masayuki Nakamoto, Ms. Misato Takigahira, Ms. Akiko Suzuki, and Ms. Naomi Maruyama for their excellent technical assistance.

This work was supported in part by Grants-in-Aid for Scientific Research from the Ministry of Education, Culture, Sports, Sci- ence and Technology of Japan (17016030) and from the Japan Society for the Promotion of Science $(21590305,24590348,15 \mathrm{k} 15063$, and 16H04697).

\section{Disclosure Statement}

The authors have no conflict of interest to disclose.

\section{References}

1 Thiery JP, Sleeman JP: Complex networks orchestrate epithelial-mesenchymal transitions. Nat Rev Mol Cell Biol 2006;7:131-142.

$\checkmark 2$ Thiery JP, Acloque H, Huang YJR, Nieto MA: Epithelial-mesenchymal transitions in development and disease. Cell 2009;139:871-890.

-3 Kalluri R, Weinberg RA: The basics of epithelial-mesenchymal transition. J Clin Invest 2009;119:1420-1428.

4 Thiery JP: Epithelial-mesenchymal transitions in tumour progression. Nat Rev Cancer 2002;2:442-454.

5 Craene BD, Berx G: Regulatory networks defining EMT during cancer initiation and progression. Nat Rev Cancer 2013;13:97-110.

-6 Lamouille S, Xu J, Derynck R: Molecular mechanisms of epithelial-mesenchymal transition. Nat Rev Mol Cell Biol 2014;15:178196.

-7 Chaffer CL, Weinberg RA: A perspective on cancer cell metastasis. Science 2011;331: 1559-1564.

8 Ikeguchi M, Miyake T, Matsunaga T, Yamamoto M, Fukumoto Y, Yamada Y, Fukuda K, Saito H, Tatebe S, Tsujitani S: Recent results of therapy for scirrhous gastric cancer. Surg Today 2009;39:290-294.

-9 Takahashi I, Matsusaka T, Onohara T, Nishizaki T, Ishikawa T, Tashiro H, Wakazugi K, Kume K, Maehara Y, Sugimachi K: Clinicopathological features of long-term survivors of scirrhous gastric cancer. Hepatogastroenterology 2000;47:1485-1488.

10 Hippo Y, Yashiro M, Ishii M, Taniguchi H, Tsutsumi S, Hirakawa K, Kodama T, Aburatani $\mathrm{H}$ : Differential gene expression profiles of scirrhous gastric cancer cells with high metastatic potential to peritoneum or lymph nodes. Cancer Res 2001;61:889-895.

$\checkmark 11$ Samel S, Singal A, Becker H, Post S: Problems with intraoperative hyperthermic peritoneal chemotherapy for advanced gastric cancer. Eur J Surg Oncol 2000;26:222-226.

12 Yanagihara K, Tanaka H, Takigahira M, Ino Y, Yamaguchi Y, Toge T, Sugano K, Hirohashi S: Establishment of two cell lines from human gastric scirrhous carcinoma that possess the potential to metastasize spontaneously in nude mice. Cancer Sci 2004;95:575-582.

13 Yanagihara K, Takigahira M, Tanaka H, Komatsu T, Fukumoto H, Koizumi F, Nishio
K, Ochiya T, Ino Y, Hirohashi S: Development and biological analysis of peritoneal metastasis mouse models for human scirrhous stomach cancer. Cancer Sci 2005;96: 323-332.

14 Ureshino H, Murakami Y, Watari K, Izumi H, Kawahara A, Kage M, Arao T, Nishio K, Yanagihara K, Kinoshita H, Kuwano M, Ono $\mathrm{M}$ : N-myc downstream regulated gene 1 (NDRG1) promotes metastasis of human scirrhous gastric cancer cells through epithelial mesenchymal transition. PLoS One 2012; 7:e41312.

15 Calin GA, Croce CM: MicroRNA-cancer connection: the beginning of a new tale. Cancer Res 2006;66:7390-7394.

16 Bartel DP: MicroRNAs: genomics, biogenesis, mechanism, and function. Cell 2004;116: 281-297.

17 Ambros V: The functions of animal microRNAs. Nature 2004;431:350-355.

18 Peinado H, Olmeda D, Cano A: Snail, Zeb and bHLH factors in tumour progression: an alliance against the epithelial phenotype? Nat Rev Cancer 2007;7:415-428.

19 Takei Y, Takigahira M, Mihara K, Tarumi Y, Yanagihara K: The metastasis-associated microRNA miR-516a-3p is a novel therapeutic target for inhibiting peritoneal dissemination of human scirrhous gastric cancer. Cancer Res 2011;71:1442-1453.

20 Takei Y, Ohnishi N, Kisaka M, Mihara K: Determination of abnormally expressed microRNAs in bone marrow smears from patients with follicular lymphomas. Springerplus 2014;3:288.

$21 \mathrm{Mu}$ P, Nagahara S, Makita N, Tarumi Y, Kadomatsu K, Takei Y: Systemic delivery of siRNA specific to tumor mediated by atelocollagen: combined therapy using siRNA targeting Bcl-xL and cisplatin against prostate cancer. Int J Cancer 2009;125:2978-2990.

22 Yuan Y, Makita N, Cao D, Mihara K, Kadomatsu K, Takei Y: Atelocollagen-mediated intravenous siRNA delivery specific to tumor tissues orthotopically xenografted in prostates of nude mice and its anticancer effects. Nucleic Acid Ther 2015;25:85-94.

23 Ishimoto T, Takei Y, Yuzawa Y, Hanai K, Nagahara S, Tarumi Y, Matsuo S, Kadomatsu K: Downregulation of monocyte chemoat- tractant protein-1 involving short interfering RNA attenuates hapten-induced contact hypersensitivity. Mol Ther 2008;16:387-395.

24 Inaba S, Nagahara S, Makita N, Tarumi Y, Ishimoto T, Matsuo S, Kadomatsu K, Takei Y: Atelocollagen-mediated systemic delivery prevents immunostimulatory adverse effects of siRNA in mammals. Mol Ther 2012;20: 356-366.

25 Takei Y, Kadomatsu K, Matsuo S, Itoh H, Nakazawa K, Kubota S, Muramatsu T: Antisense oligodeoxynucleotide targeted to Midkine, a heparin-binding growth factor, suppresses tumorigenicity of mouse rectal carcinoma cells. Cancer Res 2001;61:8486-8491.

26 Takei Y, Kadomatsu K, Itoh H, Sato W, Nakazawa K, Kubota S, Muramatsu T: $5^{\prime}$-, $3^{\prime}$-inverted thymidine-modified antisense oligodeoxynucleotide targeting midkine. Its design and application for cancer therapy. J Biol Chem 2002;277:23800-23806.

27 Takei Y, Kadomatsu K, Yuasa K, Sato W, Muramatsu T: Morpholino antisense oligomer targeting human midkine: its application for cancer therapy. Int J Cancer 2005;114:490497.

28 Takei Y, Kadomatsu K, Goto T, Muramatsu T: Combinational antitumor effect of siRNA against midkine and paclitaxel on growth of human prostate cancer xenografts. Cancer 2006; 107:864-873.

29 Koide N, Yasuda K, Kadomatsu K, Takei Y: Establishment and optimal culture conditions of microRNA-induced pluripotent stem cells generated from HEK293 cells via transfection of microRNA-302s expression vector. Nagoya J Med Sci 2012;74:157-165.

30 Fujimoto I, Takei Y: Atelocollagen-mediated siRNA delivery: future promise for therapeutic application. Ther Deliv 2014;5:369-371.

-31 Gregory PA, Bert AG, Paterson EL, Barry SC, Tsykin A, Farshid G, Vadas MA, KhewGoodall GJ: The miR-200 family and miR-205 regulate epithelial to mesenchymal transition by targeting ZEB1 and SIP1. Nat Cell Biol 2008; 10:593-601.

32 Park SM, Gaur AB, Lengyel E, Peter ME: The miR-200 family determines the epithelial phenotype of cancer cells by targeting the Ecadherin repressors ZEB1 and ZEB2. Genes Dev 2008;22:894-907. 
33 Korpal M, Lee ES, Hu G, Kang Y: The miR200 family inhibits epithelial-mesenchymal transition and cancer cell migration by direct targeting of E-cadherin transcriptional repressors ZEB1 and ZEB2. J Biol Chem 2008; 283:14910-14914.

34 Burk U, Schubert J, Wellner U, Schmalhofer O, Vincan E, Spaderna S, Brabletz T: A reciprocal repression between ZEB1 and members of the miR-200 family promotes EMT and invasion in cancer cells. EMBO Rep 2008;9: 582-589.

-35 Liu Y, El-Naggar S, Darling DS, Higashi Y, Dean DC: Zeb1 links epithelial-mesenchymal transition and cellular senescence. Development 2008;135:579-588.

36 Takeshita F, Patrawala L, Osaki M, Takahashi RU, Yamamoto Y, Kosaka N, Kawamata M, Kelnar K, Bader AG, Brown D, Ochiya T: Systemic delivery of synthetic microRNA-16 inhibits the growth of metastatic prostate tumors via downregulation of multiple cell-cycle genes. Mol Ther 2010;18:181-187.

-37 Mitra A, Mishra L, Li S: EMT, CTCs and CSCs in tumor relapse and drug-resistance. Oncotarget 2015;6:10697-10711.

38 Thibault B, Castells M, Delord JP, Couderc B: Ovarian cancer microenvironment: implications for cancer dissemination and chemoresistance acquisition. Cancer Metastasis Rev 2014;33:17-39.
39 Okugawa Y, Toiyama Y, Tanaka K, Matsusita K, Fujikawa H, Saigusa S, Ohi M, Inoue Y, Mohri Y, Uchida K, Kusunoki M: Clinical significance of Zinc finger E-box Binding homeobox 1 (ZEB1) in human gastric cancer. J Surg Oncol 2012;106:280-285

40 Yabusaki N, Yamada S, Murai T, Kanda M, Kobayashi D, Tanaka C, Fujii T, Nakayama G, Sugimoto H, Koike M, Nomoto S, Fujiwara M, Kodera Y: Clinical significance of zinc-finger E-box binding homeobox 1 mRNA levels in peritoneal washing for gastric cancer. Mol Clin Oncol 2015;3:435-441.

-41 Murai T, Yamada S, Fuchs BC, Fujii T, Nakayama G, Sugimoto H, Koike M, Fujiwara M, Tanabe KK, Kodera Y: Epithelial-to-mesenchymal transition predicts prognosis in clinical gastric cancer. J Surg Oncol 2014;109: 684-689.

42 Minn YK, Lee DH, Hyung WJ, Kim JE, Choi J, Yang SH, Song H, Lim BJ, Kim SH: MicroR NA-200 family members and ZEB2 are associated with brain metastasis in gastric adenocarcinoma. Int J Oncol 2014;45:2403-2410.

43 Dai YH, Tang YP, Zhu HY, Lv L, Chu Y, Zhou YQ, Huo JR: ZEB2 promotes the metastasis of gastric cancer and modulates epithelial mesenchymal transition of gastric cancer cells. Dig Dis Sci 2012;57:1253-1260.
44 Galván JA, Zlobec I, Wartenberg M, Lugli A, Gloor B, Perren A, Karamitopoulou E: Expression of E-cadherin repressors SNAIL, ZEB1 and ZEB2 by tumour and stromal cells influences tumour-budding phenotype and suggests heterogeneity of stromal cells in pancreatic cancer. Br J Cancer 2015;112:19441950.

45 Liu C, Yang F: Akt2/ZEB2 may be a biomarker for exfoliant cells in ascitic fluid in advanced grades of serous ovarian carcinoma. Tumour Biol 2015;36:7213-7219.

46 Notle SM, Singh SK: Origins of metastasisinitiating cells; in Srivastava R, Shankar S (eds): Stem Cells and Human Diseases. Springer Netherlands, 2012, chapt 11, pp 229-246.

47 Kelly K, Yin JJ: Prostate cancer and metastasis initiating stem cells. Cell Res 2008;18:528537.

48 Baccelli I, Trumpp A: The evolving concept of cancer and metastasis stem cells. J Cell Biol 2012;198:281-293.

49 Alix-Panabières C, Pantel K: Challenges in circulating tumour cell research. Nat Rev Cancer 2014;14:623-631.

50 Gao P, Jiao SC, Bai L, Wang H, Jing FF, Yang JL: Detection of circulating tumour cells in gastric and hepatocellular carcinoma: a systematic review. J Int Med Res 2013;41:923933
miRNAs Involved in Peritoneal Dissemination of Scirrhous Gastric Cancers 